\section{Fitness Measures and Health Outcomes in Youth}

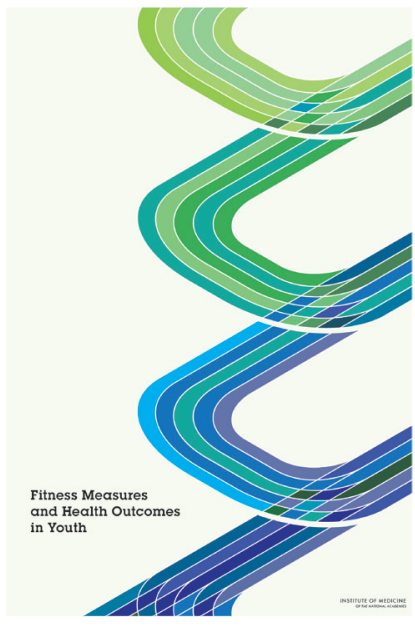

Physical fitness is a key tenant of health. It affects our ability to function and be physically active and, at poor levels, is associated with such health outcomes as diabetes and cardiovascular disease.

Physical fitness testing in American youth was established on a large scale in the 1950s. In the intervening decades, the underlying philosophies that have guided development of fitness testing protocols and specific test items used in test batteries have evolved considerably. An early focus on performancerelated fitness, measured by test items that relied heavily on power and speed, gradually gave way to an emphasis on health-related fitness, composed of components that were linked to health outcomes. Health-related fitness typically has been defined as including body composition, cardiorespiratory endurance, musculoskeletal fitness, and flexibility. Although extensive evidence links performance on specific measures of fitness to health outcomes in adults, such evidence in children and adolescents is less abundant and debate continues about the best fitness measures for youth. Using appropriately selected measures to collect fitness data in youth will advance our understanding of how fitness among youth translates into better health.

At the request of the Robert Wood Johnson Foundation, the Institute of Medicine (IOM) appointed a study committee to assess the relationship between youth fitness test items and health outcomes, recommend the best fitness test items, provide guidance for interpreting fitness scores, and provide an agenda for needed research. The IOM committee presents its findings and recommendations in Fitness Measures and Health Outcomes in Youth. For the purposes of this report, "youth" is defined as children aged 5-18.
Using appropriately selected measures to collect fitness data in youth will advance our understanding of how fitness among youth translates into better health. 


\section{Indicators for National Surveys}

As one of its primary objectives, the IOM committee was asked to identify physical fitness test items for youth that are valid, reliable, and related to health outcomes, and that would be suitable to include in national surveys to assess healthrelated fitness in youth. The committee used a systematic review conducted by the Centers for Disease Control and Prevention as its primary evidence base. The committee examined studies measuring body composition, cardiorespiratory endurance, musculoskeletal fitness, and flexibility and found varying degrees of evidence. It found that a substantial body of evidence supports specific test items that are related to health for body composition and cardiorespiratory endurance. While adequate evidence supports the relationship between musculoskeletal fitness and health, there is less evidence linking specific musculoskeletal test items to health. The evidence linking flexibility and health in youth is the least convincing. Thus, the committee recommends that national surveys of health-related fitness in youth include selected measures of body composition, cardiorespiratory endurance, and musculoskeletal fitness. (See box.)

The committee also recommends that developers of fitness test batteries use age- and genderspecific cut-points to identify individuals at risk of poor fitness-related health outcomes. A cut-point is a performance score above or below which a health risk may exist. Ideally, a cut-point is a standard value that is related to health. Often, interim values need to be used until the necessary data are available to establish health-related cut-points. The committee provides guidance for developing cut-points when all the necessary data exist, but also for developing interim cut-points when data are limited.

\section{Testing in Educational Settings}

Fitness tests are used in schools as educational tools to teach youth and their families about the

\section{BOX: Fitness Measures}

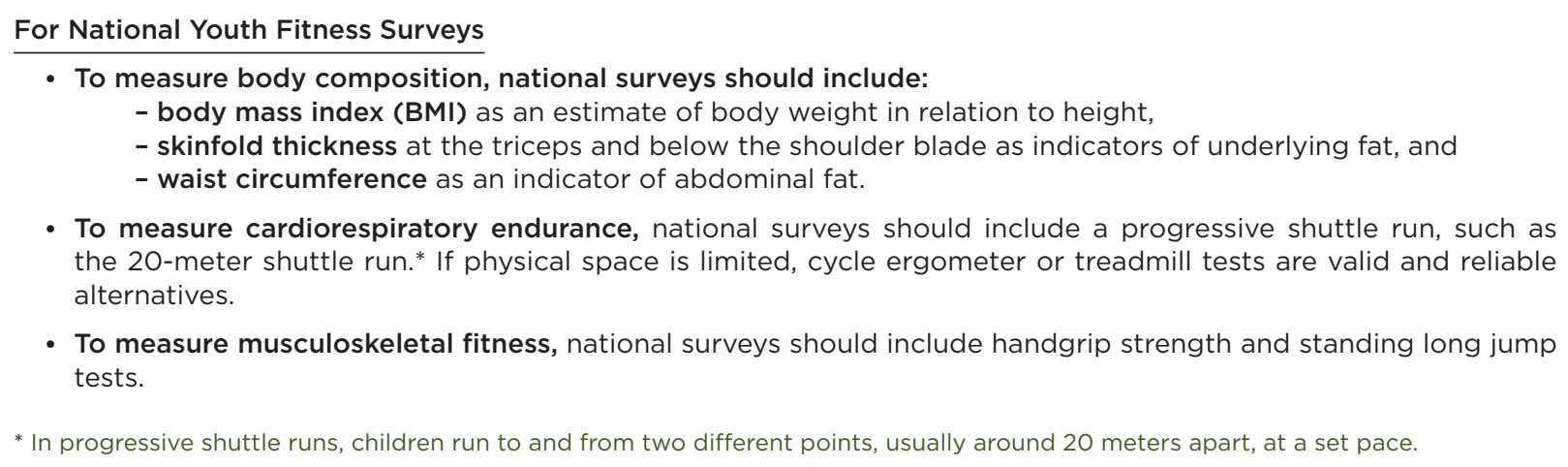

\section{For Fitness Testing in Schools}

- To measure body composition, BMI should be used.

- To measure cardiorespiratory endurance, schools should include a progressive shuttle run.

- To measure musculoskeletal fitness, schools should include handgrip strength and standing long jump tests.

For schools, additional test items that have not yet been shown to be related to health but that are valid, reliable, and feasible also may be considered as supplemental educational tools (such as distance or timed runs for cardiorespiratory endurance, the modified pull-up and push-up for measuring upper-body musculoskeletal strength, or the curl-up for measuring core strength). A measure of flexibility, such as the sit-and-reach test, also may be included. 
importance of physical fitness and to guide individuals to identify and achieve attainable goals for maintaining fitness and health. Thus, in addition to their relationship to health and practicality, the committee considered their value as an educational tool when selecting measures recommended for schools. (See box.)

School leaders and teachers should tailor test items to their schools, weighing such variables as available equipment, physical space, and availability of test administrators, as well as factors related to cost and students' privacy. For example, it might be more feasible to conduct a progressive shuttle run or a distance run as a measure of cardiorespiratory endurance in schools because they need minimal equipment compared with a cycle ergometer or treadmill.

Appropriate interpretation and communication of test results is an important element of fitness testing in schools. When school staff interpret and communicate test results to youth and their families, they should take into consideration confidentiality, self-esteem, and other sensitivities surrounding physical fitness testing. If interpreted and communicated appropriately, the results provide an opportunity to assist students in improving their health, preventing disease, and understanding fitness.

\section{Agenda for Research}

Even as fitness tests are implemented, research must continue apace. Many of the studies the committee reviewed were not designed to directly answer questions related to the relationship of fitness tests to health and presented limitations. For these reasons, the committee recommends conducting well-designed studies aimed at advancing understanding of the relationship between fitness components and health in youth.

Researchers should ensure that the exercise interventions are both specific and sufficient to improve fitness, and they should design studies that allow for analyzing the effect of complicating factors, such as nutrition, demographic variables, and maturity status.

Longitudinal studies are needed to provide scientifically sound health markers linking fitness from youth into adulthood. Collecting both fitness and health data in the same individuals would allow investigators to corroborate direct relationships between specific test items and health outcomes or markers. When an association between a fitness test and a health outcome or marker is confirmed, research should be conducted to establish and validate health-related cut-points for that test.

\section{Conclusion}

Since the first national physical fitness test for youth was developed in 1958, identifying the most appropriate measures to include has been a subject of ongoing debate. Amid a resurgence of interest in youth fitness, this IOM report concludes that selected cardiorespiratory endurance and body composition measures are related to health; are valid, reliable, and feasible; and should 
Committee on Fitness Measures and Health Outcomes in Youth

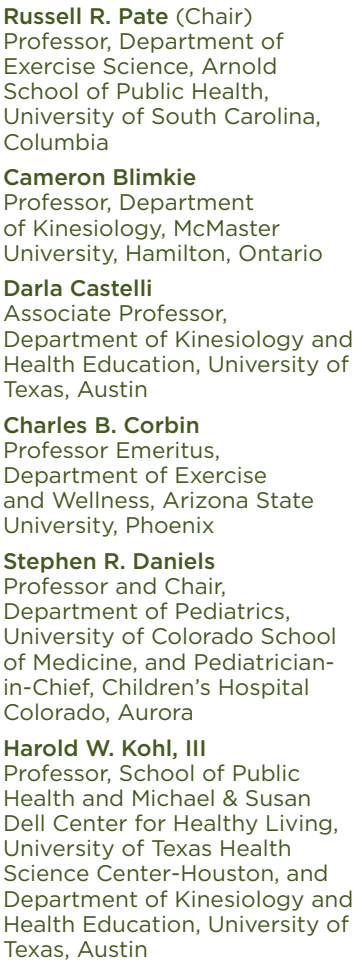

Study Staff

Maria Oria

Study Director

Laura Pillsbury

Program Officer

Allison Berger

Senior Program Assistant

Alice Vorosmarti

Research Associate

Consultants

Michael W. Beets

University of South Carolina,

Columbia

Rona Briere

Briere Associates, Inc.

Arlington, Virginia

William H. Dietz

Centers for Disease Contro

and Prevention, Atlanta,

Georgia (Retired)

Joan M. Dorn

Centers for Disease Contro

and Prevention, Atlanta,

Georgia

Janet E. Fulton

Centers for Disease Control

and Prevention, Atlanta,

Georgia

Study Sponsor

The Robert Wood Johnson Foundation
Centers for Disease Control

and Prevention, Atlanta,

Georgia

Melinda Millard-Stafford

Georgia Institute of

Technology, Atlanta

Jane Wargo

President's Council on Fitness, Sports, and Nutrition, Rockville, Maryland be included in fitness surveys and in schools. Likewise, some valid, reliable, and feasible musculoskeletal fitness measures may be related to health and also should be included in fitness batteries. Although a relationship to health was not found, a valid, reliable, and practical measure of the flexibility component might be considered in schools for educational purposes.

Collecting fitness data nationally and in schools helps with setting and achieving fitness goals and priorities for public health at an individual and national level. In addition, measuring fitness in national surveys and in schools with test items that have been demonstrated to be health-related, valid, reliable, and practical will generate data to increase our understanding of the importance of physical fitness in youth.

Due to limitations of the available research, the IOM committee recommends that data need to be collected from studies designed to answer remaining questions related to fitness tests and health in youth and to advance the national conversation about the best measures of fitness in youth.

\section{INSTITUTE OF MEDICINE}

OF THE NATIONAL ACADEMIES
Advising the nation - Improving health

500 Fifth Street, NW

Washington, DC 20001

TEL 202.334.2352

FAX 202.334.1412

www.iom.edu

The Institute of Medicine serves as adviser to the nation to improve health. Established in 1970 under the charter of the National Academy of Sciences, the Institute of Medicine provides independent, objective, evidence-based advice to policy makers, health professionals, the private sector, and the public. 